\title{
Response Analysis of Fixed-frequency Vibration Endurance Test on A Certain Airplane Oil Pressure Reservoir
}

\author{
Daqian Zhang ${ }^{1, a^{*}}$, Jinliang Zhang ${ }^{1, b}$, Zhengyang $\mathrm{Li}^{1, \mathrm{c}}$ and Qingchun Sun ${ }^{1, \mathrm{~d}}$ \\ ${ }^{1}$ Key Laboratory of Liaoning Province for Composite Structural Analysis of Aerocraft and Simulation, \\ Shenyang Aerospace University ,Shenyang 110136, China.

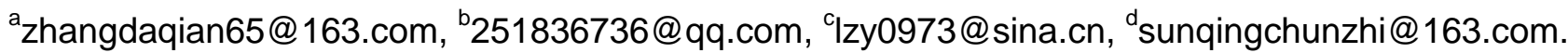

Keywords: Oil pressure reservoirs; Finite element; Fluid-solid coupling; Fixed-frequency vibration endurance test; Harmonic response;

Abstract. airplane oil pressure reservoirs are major fuel supplying parts of airplanes to have flight maneuvers. Since they are always working under alternating loadings, they are prone to produce fatigue fractures. Therefore, they will undergo vibration endurance tests in general. And the fixed-frequency vibration endurance test is commonly used in engineering. This paper has established a finite element model for the vibration test system, taken into consideration the influence of liquid fuel and introduced the fluid-solid coupling method to conduct harmonic response analysis on different excitation conditions with MSC.Nastran, thus obtaining the stress distribution under different loading conditions. Moreover, the results have been compared with the test results to verify correctness of the method, thus providing references for the improvement in structural design.

\section{Introduction}

Airplane oil pressure reservoirs mainly guarantee the sustained and stable fuel supply to airplanes in inverted flight. During the flight, the oil pressure reservoir will be excited by external vibration. Combined with the coupling of internal fuel, it easily causes vibration fatigue damage in weak parts, thus resulting in cracks and fuel leakage and finally leading to flight accidents [1]. Therefore, it is of great significance to research the vibration resistance of oil pressure reservoirs.

Major test methods to simulate the vibration environment include sine sweep, sinusoidal fixed frequency, random vibration, etc [2]. Random vibration test is able to well simulate the real vibration environment of the reservoir; however, the fatigue life prediction is relatively conservative [3]. Sinusoidal vibration endurance test is used to research the determined vibration of a product at certain frequencies; the method is simple and convenient for the structure to conduct vibration endurance test at a certain resonant frequencies. Since it can better predict the vibration resistance of parts and components, it is commonly used in engineering for vibration endurance test [4].

This paper took full account of the interaction between the reservoir and the internal fuel and utilized the fluid-solid coupling method to conduct harmonic response analysis on the sinusoidal fixed frequency test system, thus obtaining the stress distribution under different test conditions. It was compared with the actual test results to determine the correctness of the calculated results, thus achieving purposes of shortening test cycles and guiding the structural design improvement.

\section{Finite Element Equations of the Coupling System}

As for the structural model of oil pressure reservoirs, discrete differential equation of motion is expressed as(Eq. 1):

$$
\left[m_{s s}\right]\{\ddot{u}\}+\left[k_{s s}\right]\{u\}=\left\{F_{s}\right\}
$$

where $\left[m_{s s}\right]$ and $\left[k_{s s}\right]$ are mass matrices and stiffness matrices of the structure; $\{u\}$ is the structural displacement array; $\left\{F_{s}\right\}$ is the loading matrix applied to the structure.

The discrete finite element equation of fluid domain of the reservoir cavity is expressed as(Eq. 2): 


$$
\left[m_{f f}\right][\ddot{p}\}+\left[k_{f f}\right]\{p\}=\left\{F^{\prime}\right\}
$$

where $\left[m_{f f}\right\rfloor$ and $\left[k_{f f}\right\rfloor$ are mass matrices and stiffness matrices of the fluid domain; $\{p\}$ is the fluid pressure array; $\left\{F^{\prime}\right\}$ is the generalized force matrix transmitted from unit surface[5].

Considering the interaction between the reservoir structure and internal fuel, the finite element equation of the coupling system is expressed as(Eq. 3):

$$
\left[\begin{array}{cc}
m_{s s} & 0 \\
\left(\rho_{0} c_{0}\right)^{2} S^{T} & m_{f f}
\end{array}\right]\left\{\begin{array}{l}
\ddot{u} \\
\ddot{p}
\end{array}\right\}+\left[\begin{array}{cc}
k_{s s} & -S \\
0 & k_{f f}
\end{array}\right]\left\{\begin{array}{l}
u \\
p
\end{array}\right\}=\left\{\begin{array}{l}
F_{s} \\
0
\end{array}\right\}
$$

where $[S]$ is the fluid-solid coupling matrix; $c_{0}$ is the speed of transmission of sound in the fuel; $\rho_{0}$ is the fuel density.

\section{Establishment of the Finite Element Model of Fluid-solid Coupling System}

It's a basic premise of vibration fatigue analysis to obtain stress distribution of the structure at different frequencies via finite element methods. Middle surfaces of the outer wall, reinforcing plates, tube joints, supporting arms, bandages and other thin parts were extracted without influencing the stress of the reservoir and simulated with shell elements; the base was simulated with tetrahedral elements because of its thickness; the shock isolation rubber was simulated with hexahedral elements.

Coarse meshes with larger dimensions than those of the external structures were employed for acoustic fluid elements. For incompressible fluids, however, the influence of quality was more important. At this time, meshes of fluids should be equivalent to structured meshes. Moreover, for fluid elements, there was no particular accuracy advantage of hexahedral elements [6]. ANSA pre-processing software was utilized to generate tetrahedral fluid elements via shell elements of the reservoir wall. Coordinates of the spatial positions of fluid and solid nodes matched with each other on the fluid-solid coupling surface.

The bolt was simulated with MPC cells and the welding seam between the reinforcing plate and the reservoir wall was connected with corresponding rigid elements RBE2 to the node. The reservoir was connected with the bandage and the supporting arms with ANSA to quickly generate RBE3-HEX-RBE3, hereby realizing flexible connections between the hexahedron elements and both shell elements. The ultimate finite element model containing fluids is shown in Figure 1. The basic length of the element is $10 \mathrm{~mm}$, with a total of 372190 elements and 102468 nodes.

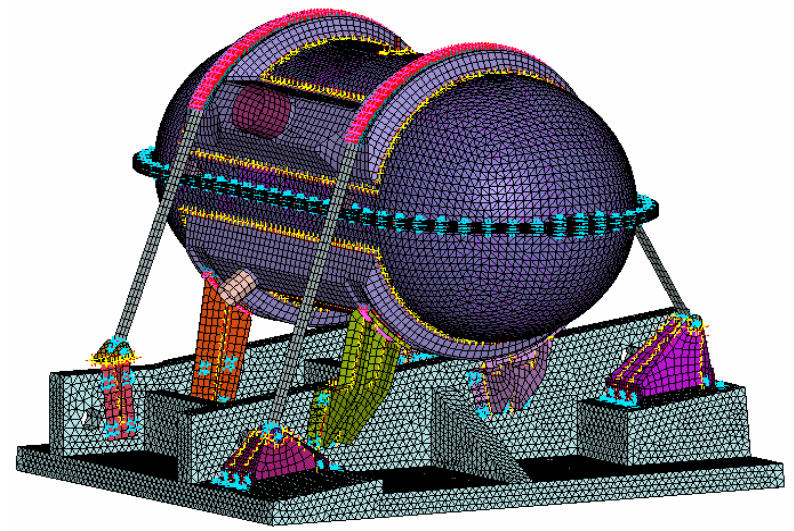

Figure 1 A Finite Element Model with Liquid Fuel 


\section{Harmonic Response Analysis of the Vibration System}

Since it is difficult to solve the long time transient response with finite element, the efficient frequency-domain analysis method was utilized to conduct harmonic response analysis, so as to obtain dynamic response of the reservoir.

The base was fixed on the test bench to facilitate loading. All nodes of the bottom surface of the base were crawled up via RBE2 elements; the degree of freedom of these nodes were coupled to the master node of RBE2; dynamic excitation was imposed on the master node in accordance with the test loading spectrum (Table 1); the excitation was evenly distributed to the base. With regard to loading frequencies, displacement excitation was adopted for low loading frequencies while acceleration excitation was employed for high loading frequencies. Since the types of excitation were different and frequencies were disperse, Nastran sol108 Direct Method was utilized to solve harmonic response [7], so as to obtain the stress distribution under different test conditions.

In order to verify correctness of the simulation results, harmonic response analysis results of the reservoir were compared with the test results (Table 1); the finite element model had high accuracy. Dynamic response of the oil pressure reservoir achieved the maximum value at $100 \mathrm{HZ}$ and $144 \mathrm{HZ}$ : about 50MPa, in both supporting arms. (Figure 2-3)

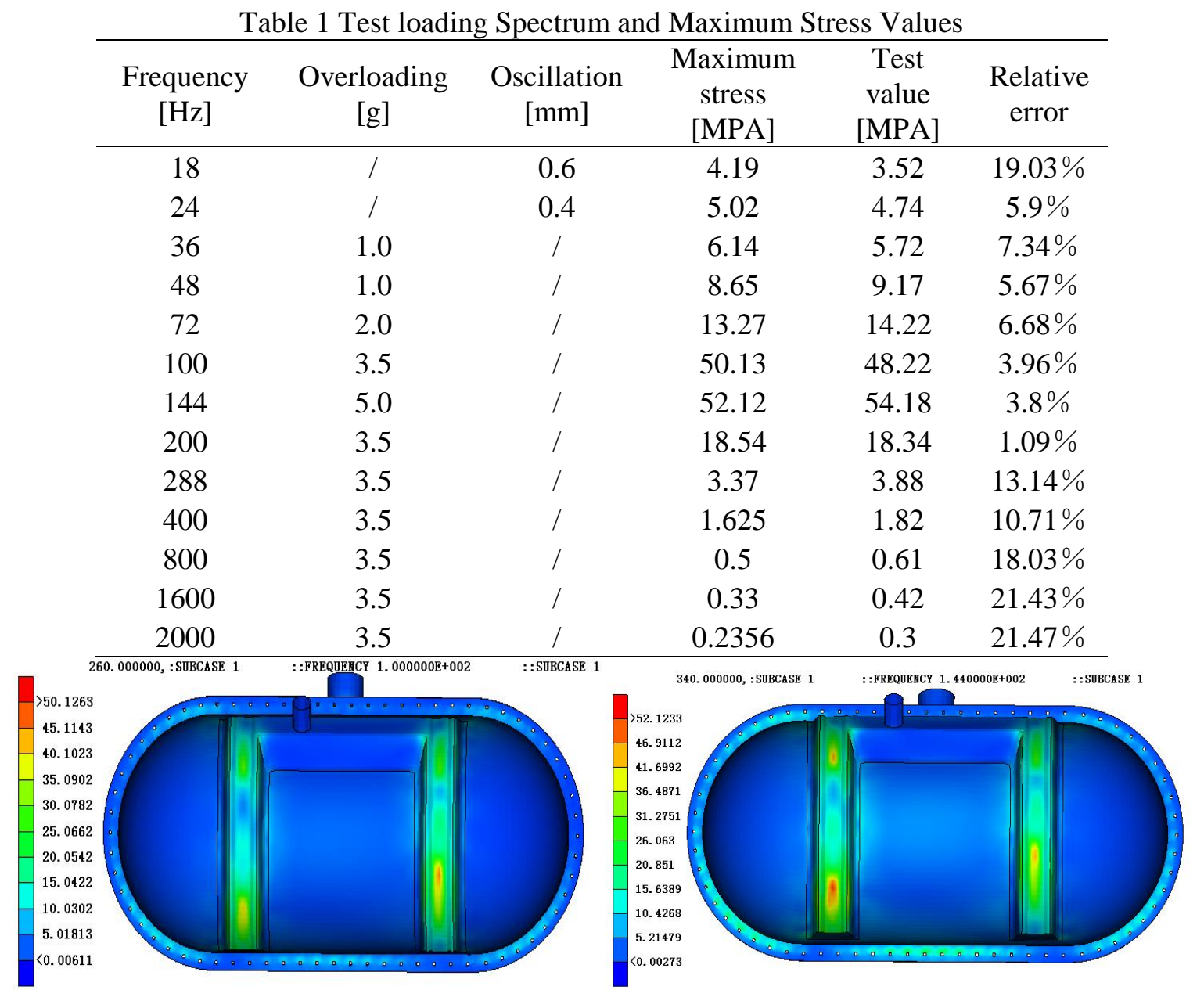

Figure 2 Stress of the Reservoir at $100 \mathrm{HZ}$

Figure 3 Stress of the Reservoir at $144 \mathrm{HZ}$

\section{Results Analysis}

It can be seen from the calculated results (Table 1): maximum stress of the reservoir gradually increases with the excitation frequencies and then decreases; the overall stress level of the oil pressure reservoir is low. The maximum stress value $(52.12 \mathrm{MPa})$ appears at $144 \mathrm{HZ}$; the oil pressure reservoir material is LF21, with a yield stress of 70MPa. The maximum stress value is close to the yield stress of the material. Therefore, further fatigue life analysis is required to verify its vibration strength [8]. 
The maximum stress of the reservoir under other load cases is much smaller than the yield stress (70MPa); the vibration strength is enough.

\section{Conclusion}

(1) This paper adopts the fluid-solid coupling method to take into account the influence of fuel and conducts harmonic response analysis on the coupling system. Since the dynamic stress obtained via simulation well coincides with the test results, it is proved that the fluid-solid coupling method utilized in this paper is feasible; it is able to be used for simulation analysis for vibration test of the oil pressure reservoir.

(2) Maximum stress of the reservoir varies with excitation frequencies; the stress level is high in medium-frequency band and low in low-frequency and high-frequency ranges.

(3) Dynamic stress of the reservoir is close to its yield limit when the excitation frequency is 144HZ. Therefore, further fatigue life analysis is required to analyze its vibration strength.

(4) Conducting simulation analysis during the design phase with the analysis method presented in this paper will improve efficiency and save costs.

\section{References}

[1] Keran Wang, Guangshuang Yang, Shubo Li, et al. Aircraft Design, 2005, (1): 20-25. (In Chinese)

[2] Kai Yan, Qingjun Song, Qiuxiang Sun, et al. Laborratory Science, 2013, 16(6): 43-44. (In Chinese)

[3] Zhao Zhang, Wanyu Zhang, Yaqi Hu. Aeronautical Computing Technique, 2012, 42(2): 60-64. (In Chinese)

[4] Li Chen. Equipment Environmental Engineering, 2013, (2): 48-51. (In Chinese)

[5] Tianfei Ma, Yi Lin, Jianwei Zhang. Chinese Journal of Mechanical Engineering, 2005, 41(7): 225-230. (In Chinese)

Reference to a book:

[6] MSC/NASTRAN BASIC DYNAMIC ANALYSIS, The MSC.Software Corporation, (2014) 434-437.

[7] Livne E, Blando G D. Aiaa Journal, 2012, 41(7): 1377-1385.

Reference to a book:

[8] Chuanyue Zhou, Hongxia Zheng. MSC.Fatigue Analysis Application and Example. BeiJing: Science Press, 2005. (In Chinese) 